\title{
Bidimensional modeling for incompressible viscous flow using the Circumcenter Based Approach in an unstructured grid
}

\author{
A. L. Fazenda ${ }^{1}$ \& J. S. Travelho ${ }^{2}$ \\ ${ }^{1}$ Department of Science and Technology, \\ Federal University of São Paulo, São José dos Campos-SP, Brazil \\ ${ }^{2}$ Computing and Applied Mathematics Laboratory, \\ National Institute for Space Research, São José dos Campos-SP, Brazil
}

\begin{abstract}
A possible approach for modeling two-dimensional convection-diffusion problems in a cell-centered scheme with an unstructured triangular grid is the use of the Circumcenter, that is the center of the circumference that passes through the vertices of the triangular volume. This point is used to calculate all variables involved in the numerical simulation, and a Finite Volume Method was use to discretize the equations of an Incompressible Viscous Flow. This work analyzes classical problems of bidimensional flow, such as the inlet region of a Poiseuille flow, lid-driven cavity, backward-facing step and free convection with Boussinesq approximation. The application of the method has been shown to be a simple and flexible scheme and the results fit the analytical, experimental or numeric data presented in the literature.
\end{abstract}

Keywords: unstructured grid, cell-centered scheme, incompressible flow, Circumcenter Approach.

\section{Introduction}

The numerical modeling of incompressible viscous flow has received special attention in recent decades, in particular because of the Velocity-Pressure relationship. Therefore, numerous different strategies to create equations that model the phenomena have been developed. 
In this work, a co-located cell-centered scheme in the primitive variables is developed, under a triangular unstructured grid. The viscous term of the NavierStokes equation is discretized with the "Circumcenter Based Approach" (CBA) [9], and an up-wind scheme is used in the convective term.

The pressure field is calculated with an artificial equation, created by applying a divergent operator in the Momentum equation, and considers Neumann boundary conditions. The final algorithm is easy to use and understand, without equations to correct or under-relax any of the primitive variables used. The Linear System Equations involved are calculated by a specific solver, according to the form of the matrix resulting.

Computational benchmarks were realized in classical 2D flow problems, considering an incompressible, viscous and laminar flow. The problems are: flow under flat plates (Poiseuille Flow), Lid-driven cavity flow, suddenly expansion in a step (Backward-facing step) and natural or free convections problems (with Boussinesq modeling approach).

\section{Basic equations}

The equations used, in this document, for the numerical simulation of an uncompressible flow in steady state, will be:

$$
\begin{gathered}
\nabla \cdot(\rho \vec{V})=0, \\
\rho \nabla \cdot(\vec{V} \vec{V})=\mu \nabla^{2} \vec{V}+\rho \vec{g}-\nabla P, \\
\nabla \cdot(\rho \vec{V} T)=\nabla \cdot\left(\frac{k}{c_{P}} \nabla T\right),
\end{gathered}
$$

The equation (1) represents the continuity equation; the equation (2) represents the momentum equation and (3) represents the energy equation. In above equations, the symbol $\rho$ defines the fluid density; the vector $\vec{V}$ defines the velocity vector; $\mu$ represents the viscosity; $P$ represents the Pressure field; $T$ defines the temperature; $\kappa$ the thermal conductibility and $C_{p}$ the specific heat at constant pressure.

It is important to mention that in all numerical simulations presented in this paper we consider an incompressible laminar flow with constant density and viscosity.

In the case of free convection, the flow is not isothermal; however, this temperature buoyancy should not cause significant changes in density and viscosity of the fluid.

\section{The relationship between velocity and pressure in incompressible flows}

In incompressible flows, there is not an explicit equation to calculate the pressure evolution, and it is common to use the momentum and continuity equations to derive a new equation to determine the required pressure values. 


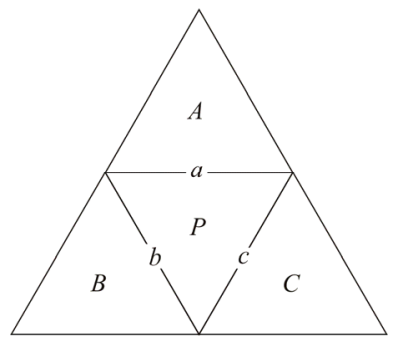

Figure 1: $\quad$ Stencil for the calculation of each triangular element.

In this case, primitive variables with a Poisson equation will be used to allow pressure determination, following Gresho and Sani [1]. It can be obtained through the application of a divergent operator in the momentum equation (2). After some rearrangements, it can be defined as:

$$
\nabla^{2} P=\nabla \cdot\left[-\rho \nabla \cdot(\vec{V} \vec{V})+\mu \nabla^{2} \vec{V}+\rho \vec{g}\right]
$$

So, the equations to be used in the numerical simulation will be: (1), (2) and (4). For non-isothermal cases the equation (3) must be added and solved also.

\section{Circumcenter Based Approach (CBA)}

To discretize the partial differential equations defined by (1), (2), (3) and (4) the Finite Volume Method [10] will be used. With the unstructured triangular grid used, it is necessary to integrate each PDE of the problem in the triangular cell. Considering the stencil in figure 1 , each central control volume will be called " $P$ " and the three neighbors' cells will be called $A, B, C$. The cell faces for $P$ will be defined $a, b, c$, and each of them share the $P$ cell with $A, B$ and $C$ cell, respectively.

The resultant discretized equation for (2) will be:

$$
\begin{aligned}
\rho \oint_{S}(\hat{n} \cdot \vec{V}) \vec{V} d S= & \mu \oint_{S} \hat{n} \cdot(\nabla \vec{V}) d s+\rho \vec{g} A- \\
& \left(\hat{n}_{a} P_{a} S_{a}+\widehat{n}_{b} P_{b} S_{b}+\widehat{n}_{c} P_{c} S_{c}\right)
\end{aligned}
$$

where $n_{x}$ define the normal vector to face $x, S_{x}$ define the length of face $x$ and $A$ is the area of the cell $P$.

The solution for diffusive problems in structured grid is very simple because the cell faces are perpendicular to the line linking the two neighbor cell centers. These characteristics, generally, are not found in a triangular unstructured grid. The use of a vertex centered scheme with Voronoi Diagrams causes a return to this facility.

Another way to maintain these characteristics in triangular unstructured grids is the use of CBA [9], i.e., the use of the circumcenter of each triangular cell like the center of the element. Remembering that the center of a circle or sphere that 
touches each cell vertex is the point where the respective mediatrices crosses each other. So the term $\oint_{S} \widehat{n} \cdot(\nabla \vec{V}) d s$ can be approximated by:

$$
\oint_{S} \hat{n} \cdot(\nabla \vec{V}) d s=\sum_{i=a, b, c} \frac{\partial(\vec{V})}{\partial n_{i}} S_{i} \cong \sum_{i=a, b, c} \frac{\vec{V}_{i_{i}}-\vec{V}_{P}}{d_{i}} S_{i}
$$

where $d$ defines the distance between the center of cell $P$ and the neighbor $X$. For the convective term, an upwind scheme has been used.

\section{Boundary conditions}

\subsection{Velocity with the Dirichlet condition}

In this first case the velocity on face " $a$ ", of the triangular cell, in the boundary will be predefined:

$$
\vec{V}_{a} \cdot \widehat{n}_{a}=C
$$

A special and very common case, of Dirichlet condition, to be noticed is the no slip condition:

$$
\vec{V}_{a} \cdot \widehat{n}_{a}=0
$$

In both situations the convective contribution of face " $a$ " is defined like a constant or null, respectively to (7) and (8). The pressure at the boundary, with no slip condition (8), can be considered to be constant. In the case of non-null value (7) the pressure at the boundary can be approximated in another way.

\subsection{Null velocity gradient (Neumann condition)}

This kind of boundary condition is applied specially in the outlet of a HagenPoiseuille flow. Assuming that the face " $a$ " of a cell is at the boundary, we have:

$$
\left.\frac{\partial \vec{V}}{\partial n}\right|_{a}=0
$$

In this case, the value of the velocity vector in the center of $A, B$ or $C$ cell, is equal to the value in the cell center $P$. The value of the pressure in the boundary can be determined by an extrapolation in the adjacent cells.

\subsection{Adiabatic boundary with the no slip condition}

The first derivate of $T$ in the face " $a$ " of a cell will be null:

$$
\left.\frac{\partial T}{\partial n}\right|_{a}=0
$$


The condition defined in equation (8) will be applied. The contribution of the diffusive term in Energy equation (3) for the face " $a$ " will be null, and, because of (8), the contribution of the convective term in (3) will be null too.

\subsection{Boundary with predefined temperature and the no slip condition}

The value of temperature at the boundary is predefined:

$$
T_{a}=C
$$

The condition defined in equation (8) must be applied like in the previous case.

\section{Results}

In this section the result for the application of Circumcenter Based Approach for Poiseuille flow, lid-driven cavity flow, backward-facing step flow and free or natural convection are presented.

\subsection{Poiseuille flow}

The results presented here were obtained solving an incompressible laminar viscous flow in steady state considering isothermal conditions. Brodkey [2] called the flow in a channel formed by two flat plates by Poiseuille flow, and in this case, in a fully developed flow, the maximum of the velocity parallel to the plates are obtained in the middle of the boundary distance, and corresponds to $150 \%$ of the medium velocity.

The two dimensional domain can be viewed in figure 2 .

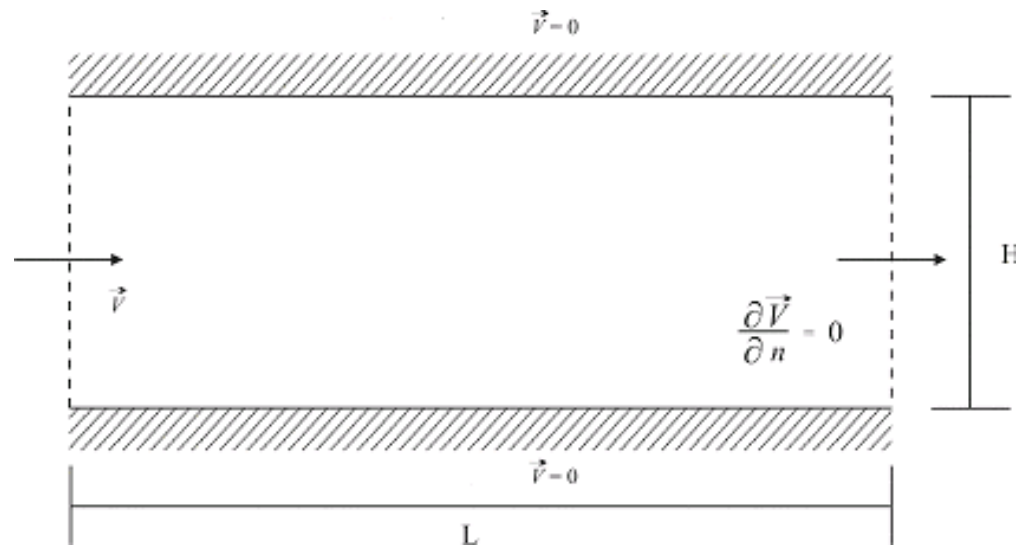

Figure 2: $\quad$ Poiseuille flow domain. 


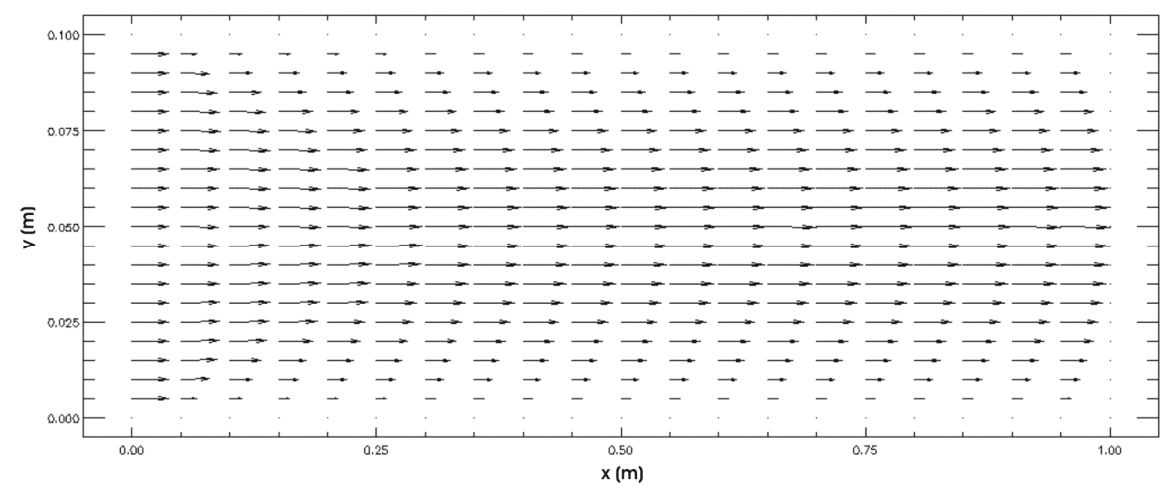

Figure 3: $\quad$ Flow for $\mathrm{Re}=100$.

The length $L$ is 10 times larger than the height $H$. The triangular unstructured grid uses 18864 vertex and 36846 triangular cells. The velocity in the inlet of the domain is a flat profile. The result showing the velocity vector for Reynolds 100 (fully developed) can be viewed in figure 3 .

The position where the fluid can be considered as fully developed $(\partial \vec{V} / \partial x \cong 0)$ depends upon the Reynolds number. For simulations with Reynolds 400 and 800 the flow is not fully developed at the outlet of the domain. In Schlichting [3] is defined a simple equation to estimate the length of the developing area $(L e)$ :

$$
\frac{L e}{H}=0.04 \mathrm{Re}
$$

The simulation shows that for Reynolds 100 the relative medium value for $\partial \vec{V} / \partial x$ is lower than $0.1 \%$ in the cell with the longitudinal coordinate is located after $0.35 \mathrm{~m}$ and for Reynolds 200 the same value is obtained for $0.75 \mathrm{~m}$, which agrees with (12).

\subsection{Lid-driven cavity flow}

In this case the interior of a cavity is filled with fluid and, in the upper boundary, there is a layer of the same fluid moving with parallel uniform velocity. The $2 \mathrm{D}$ domain, with equal length $L$ and height $H$, can be viewed in figure 4 .

In figure 5 it is possible to see three vortexes in the streamlines visualization for Reynolds 1000 .

Using Ghia et al. [4] to compare the results of the method, it is possible to see in figures 6 and 7, the velocity profile at the line that crosses $x=L / 2$ and $y=$ $H / 2$, for Reynolds 100 and 1000, respectively. Remembering that the data in the lines $x$ and $y$ are obtained with a linear interpolation. 


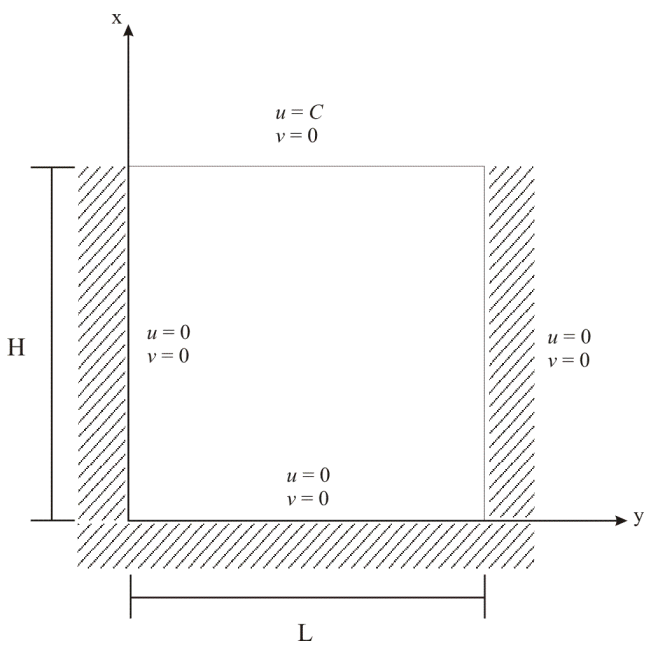

Figure 4: Lid-driven cavity flow domain.

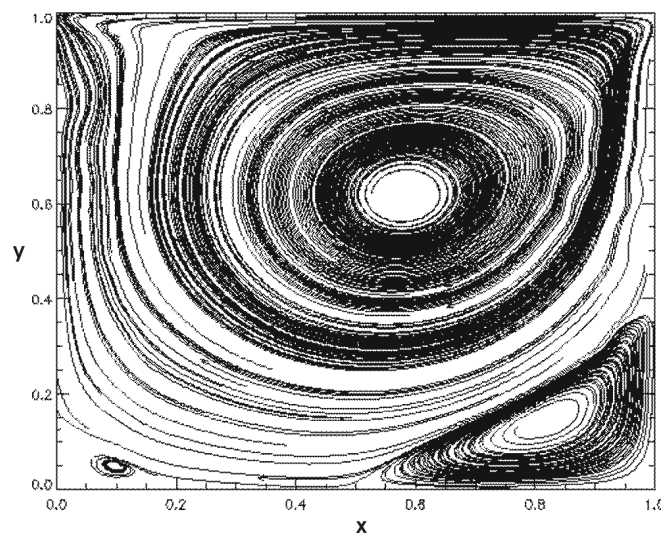

Figure 5: $\quad$ Streamlines for Reynolds 1000 in a lid-driven cavity.

\subsection{Backward-facing step flow}

This problem consists of a flow with inlet in a straight channel (height $h$ ) that suddenly opens in a larger channel (height $H$ ). The relationships between the different heights are: $H / h=2$ and the length $L$ will be $2 H$. The tests are performed with Reynolds 150, and the direction of the velocity vector can be viewed in figure 8 :

In Zhao and Zhang [12] it is possible to found physical and numerical results for Reynolds 150. The location of the main vortex after the step and the reattachment point in [13] experimental results can be compared with the numerical data obtained in this work in table 1. 


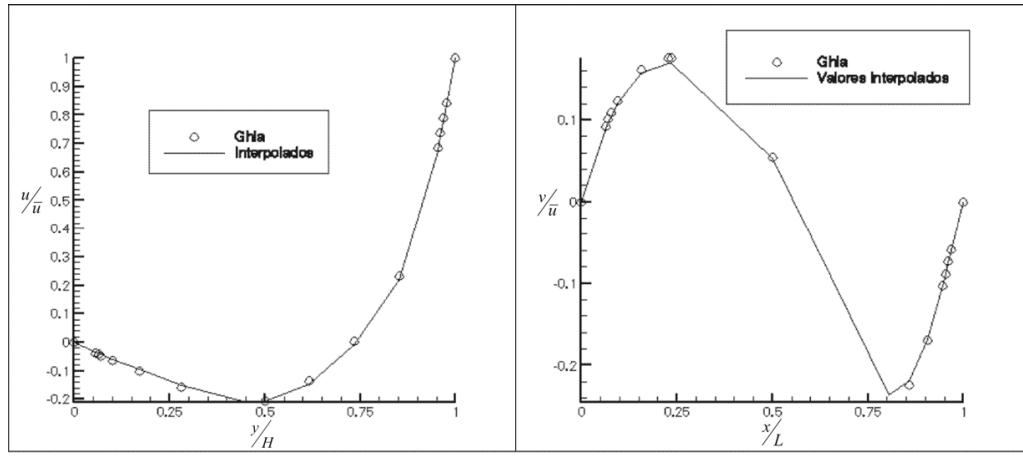

(a)

(b)

Figure 6: Velocity profile interpolated at $x=L / 2$ (a) and $y=H / 2$ (b) for Reynolds 100.

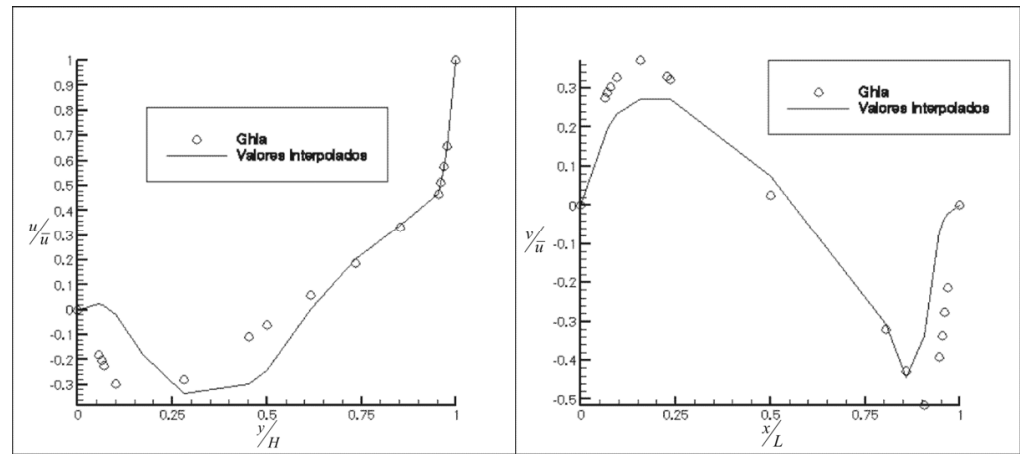

(a)

(b)

Figure 7: Velocity profile interpolated at $x=L / 2$ (a) and $y=H / 2$ (b) for Reynolds 1000.

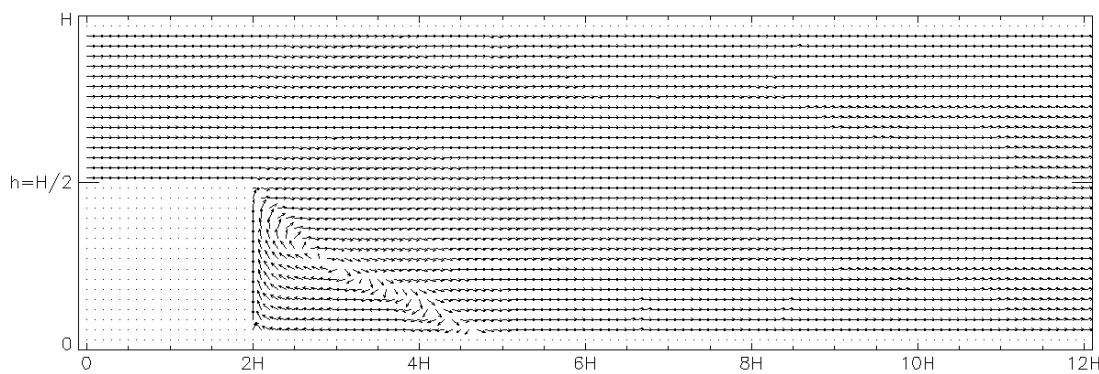

Figure 8: Direction of the velocity vector in Reynolds 150. 
Table 1: Location of the main vortex and re-attachment point.

\begin{tabular}{|l|l|l|l|}
\cline { 2 - 4 } \multicolumn{1}{c|}{} & $\begin{array}{l}\text { X coordinate } \\
\text { for vortex }\end{array}$ & $\begin{array}{l}\text { Y coordinate } \\
\text { for vortex }\end{array}$ & $\begin{array}{l}\text { Re-attachment } \\
\text { point }\end{array}$ \\
\hline Zhao \& Zhang [12] & $0,925 \mathrm{H}$ & $0,29 \mathrm{H}$ & $2,25 \mathrm{H}$ \\
\hline CBA & $0,9 \mathrm{H}$ & $0,24 \mathrm{H}$ & $2,6 \mathrm{H}$ \\
\hline
\end{tabular}

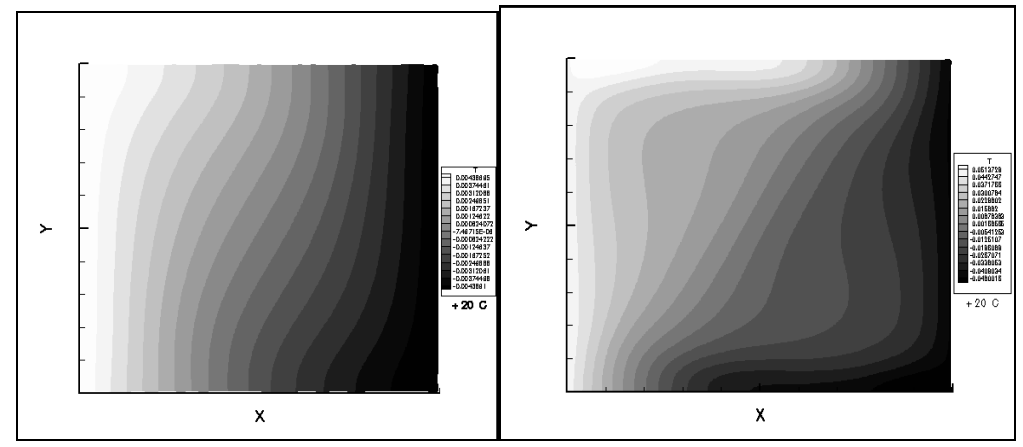

(a)

(b)

Figure 9: $\quad$ Temperature field for $\mathrm{Ra}=10^{3}(\mathrm{a})$ and $\mathrm{Ra}=10^{4}(\mathrm{~b})$.

Table 2: Nusselt number in free convection.

\begin{tabular}{|l|l|l|}
\cline { 2 - 3 } \multicolumn{1}{c|}{} & $\mathrm{Ra}=10^{3}$ & $\mathrm{Ra}=10^{4}$ \\
\hline Vahl Davis [5] & 1,116 & 2,234 \\
\hline Actual simulation & 1,06005 & 2,01911 \\
\hline
\end{tabular}

\subsection{Free or natural convection}

The main characteristics of free convection are a natural flow of the fluid because of density buoyancy when submitted to temperature or pressure variations. In this problem the density buoyancy occurs because of temperature gradient in a bidimensional domain with a box shape, where the horizontal boundaries are adiabatic, the right vertical boundary is consider hotter than medium temperature and the left vertical boundary is colder than the same reference value, and remembering that the Boussinesq approximation for the body force $\rho g$ is used in momentum equation.

The shaded graphic of temperature field for Rayleigh number (Ra) equal $10^{3}$ and $10^{4}$ can be checked in figures 9 (a) and 9(b).

Table 2 allows checking the Nusselt number calculated by the current method and the Nusselt number numerically calculated by Vahl Davis [5].

\section{Conclusions}

The Pereira Filho [13] work is the first use of CBA to solve incompressible flow problems, however, in his last work, only the Hagen-Poiseuille flow are solved. 
The 3D grid used was triangular and unstructured in $\mathrm{x}$ and $\mathrm{y}$ directions, and structured in the $\mathrm{z}$ direction (the direction of the main stream). In this work there is no structured grid in any direction and it is possible to notice recirculation vortices in almost of cases tested, including a new variable (temperature) calculation in one of them (free convection).

Analyzing the results presented, it is possible to conclude that the CBA can be used in an alternative way to modeling 2D incompressible laminar flow problems under triangular unstructured grids. The resulting discrete equations, in primitive variables, are easy to obtain and understand, without use of staggered grids, corrections or under-relax procedures.

Because of simplicity, the CBA method presents a small algorithm complexity and a low computational costs when comparing other methods used with the same purpose and characteristics, like Frink [6], Mathur and Murthy [7], Despotis and Tsangaris [8], because needs a minor arithmetic operations per "timestep".

The benchmarks performed shows promising behavior, agreeing with classical 2D incompressible problems. However, numerical instabilities were found with flows with higher Reynolds Numbers. The future inclusion of a turbulent modeling scheme in CBA needs to be investigated with this kind of 2D flows. Another problem encountered shows that the method is grid dependent, needing efficient grid generators, specially to prevent the generation of triangular element with internal angles bigger than $90^{\circ}$, like depicted in Date [11]. In this case, Delaunay triangulations minimize the problem.

This kind of problem (triangular element with internal angles bigger than $90^{\circ}$ ) makes the circumcenter be localized outside the control volume. However, the problem affects also Voronoi schemes (Vertex centered), creating polygons with sides that do not cross triangular faces. In 3D flows similar problems can occurs with tetrahedral elements (circumsphere localized outside the control volume).

For the future the authors suggests a better investigation of 3D case (preliminary analyzes shows numerical precision lose), and comparative tests with other classical approaches, like Voronoi schemes and Frink [6] scheme. A compressible modeling case and an inclusion of turbulent scheme can be explored too.

\section{References}

[1] Gresho, P.M.; Sani, R.L. On pressure boundary conditions for the incompressible Navier-Stokes equations. International Journal for Numerical Methods in Fluids, v. 7, p. 1111-1145, 1987.

[2] Brodkey, R.S. The phenomena of fluid motions. Massachusetts: AddisonWesley, 1967. $737 \mathrm{p}$.

[3] Schlichting, H. Boundary layer theory. 7. ed. New York: McGraw-Hill, 1979. $817 \mathrm{p}$.

[4] Ghia, U.; Ghia, K.N.; Shin C.T. High-Re solutions for incompressible flow using the Navier-Stokes equations and a multigrid method. Journal of Computational Physics, v. 48, p. 387-411, 1982. 
[5] Vahl Davis, G. Natural convection of air in a square cavity: a bench mark numerical solution. International Journal for Numerical Methods in Fluids, v. 3, p.249-264, 1983.

[6] Frink, N.T. Recent progress toward a three-dimensional unstructured Navier-Stokes flow solver, Reno: NASA Langley Research Center, 1994. AIAA Paper 94-0061. 24 p.

[7] Mathur, S.R.; Murthy, J.Y. A pressure-based method for unstructured meshes. Numerical Heat Transfer, Part B, v. 31, n. 2, p. 195-215, 1997.

[8] Despotis, G.K.; Tsangaris, S. Fractional step method for solution of incompressible Navier-Stokes equations on unstructured triangular meshes. International Journal for Numerical Methods in Fluids, v. 20, p. 12731288, 1995.

[9] Travelho, J.S.; Fazenda, A.L.; Enari, E.H. Abordagem pelo circuncentro para malhas não estruturadas em transferência de calor (The Circumcenter Based Approach for unstructured grid in heat transfer). In: Congresso Brasileiro de Engenharia Mecânica (Brazilian Congress in Mechanical Engineering), 15., Águas de Lindóia, 1999. Proceedings.

[10] Patankar, S.V. Numerical heat transfer and fluid flow. New York: Hemisphere, 1980. 197 p.

[11] Date, A.W. Solution of transport equations on unstructured meshes with cell-centered colocated variables. Part I: Discretization. International Journal of Heat and Mass Transfer, V. 48, p. 1117-1127, 2005

[12]Zhao, Y.; Zhang, B. A high-order characteristics upwind FV method for incompressible flow and heat transfer simulation on unstructured grids. Computational Methods Applied Mechanics Engineering, v. 190, p. 733756, 2000.

[13] Pereira Filho, N. Simulação de escoamentos incompressíveis tridimensionais com uso da abordagem baseada no circuncentro (Tridimensional incompressible flow simulation with circumcenter based approach). São José dos Campos. 126 p. PHD Thesis (Applied Computing) - National Institute for Space Research, 2000

\section{Acknowledgement}

The author, Alvaro L. Fazenda, was partially supported by FAPESP (Sao Paulo Research Foundation). 